\title{
An empirical approach for space- based mirrors to evaluate the extent of radiation compaction under expected environmental doses
}

A. Carré, M. Valente, T. Hull, J. Krieg, T. Westerhoff

A. Carré, M. Valente, T. Hull, J. Krieg, T. Westerhoff, "An empirical approach for space-based mirrors to evaluate the extent of radiation compaction under expected environmental doses," Proc. SPIE 11852, International Conference on Space Optics - ICSO 2020, 118524 U (11 June 2021); doi:

10.1117/12.2599816

SPIE Event: International Conference on Space Optics - ICSO 2021, 2021, Online Only 


\section{International Conference on Space Optics-ICSO 2020}

Virtual Conference

30 March-2 April 2021

Edited by Bruno Cugny, Zoran Sodnik, and Nikos Karafolas
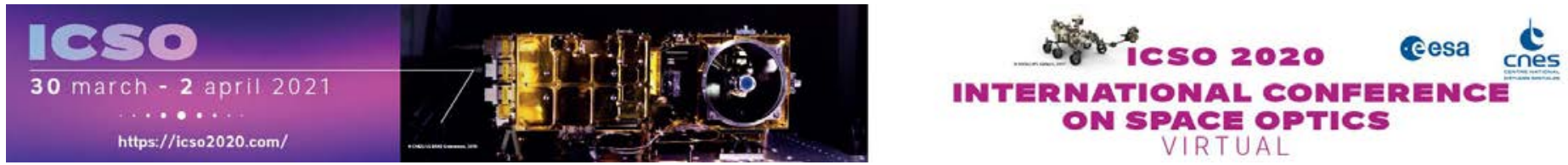

\section{An empirical approach for space-based mirrors to evaluate the extent of radiation compaction under expected environmental doses}

\section{Cesa issopoeseatings lenes}




\title{
An empirical approach for space-based mirrors to evaluate the extent of radiation compaction under expected environmental doses
}

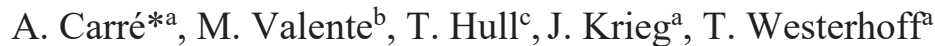 \\ ${ }^{a}$ Schott AG Mainz, Hattenbergstraße 10, 55122 Mainz, DE; \\ bArizona Optical Systems 5575 S. Houghton Rd., Tucson AZ 85747; \\ 'University of New Mexico, 21 Algodon Lane Placitas, NM 87043 USA
}

\begin{abstract}
ZERODUR $^{\circledR}$, a glass-ceramic by SCHOTT, has a successful 50-year heritage of stability in critical mirror substrates of astronomical payloads used in various orbits. This heritage includes the Hubble Space Telescope (secondary mirror) that celebrated its 30 year-in-space anniversary, and the Chandra Great Observatory (all mirrors). Although ZERODUR ${ }^{\circledR}$ exhibits a strong record of dimensional stability under orbital ionizing radiation, prior dosage measurements exhibit considerable disagreement on the magnitude of dimensional change under irradiation, and extrapolation to the level of realistic dosages presents large uncertainty. This has resulted in users of ZERODUR ${ }^{\circledR}$ needing to apply large error budget allocations for radiation effects. Our intent is to conduct rigorous measurements, matched to compaction theory, and extend measurement into lower doses realistic to mirrors in telescopes. Detailed models have been constructed, coupons designed in collaboration with the irradiation facility of the metrological institute Physikalisch-Technische Bundesanstalt (PTB) and precision optical metrology defined by Arizona Optical Systems (AOS). We will describe the models, the coupons manufacturing, the irradiation plan, and the plan defining measurement of curvature and shape change due to irradiation. The measurement data will be reconciled against the FEM results to refine accuracies in modeling. Accurate measurement in a stable set-up enables quantitative results at realistic dosages.
\end{abstract}

Keywords: ionizing radiation, telescope, satellite, ageing.

\section{INTRODUCTION}

Form stability of spaceborne optic is a crucial prerequisite needed to ensure the best possible image rendering. To this end low thermal expansion glass and/or ceramics are preferred for the manufacturing of these optics. Thus, the influence of local temperature gradients (resulting from e.g. periodic sun exposure) have only a minimal impact on the optical figure. Temperature variation is not the only root cause of deformations, effect such as compaction i.e. overall material shrinkage, induced by ionizing radiation are also mentioned. Independent investigations of radiation-induced compaction performed on the optical glass ceramic ZERODUR ${ }^{\circledR}$ led to very different results, differences by approximatively one order of magnitude have been observed between different authors, see [1][2][3][4][5]. 


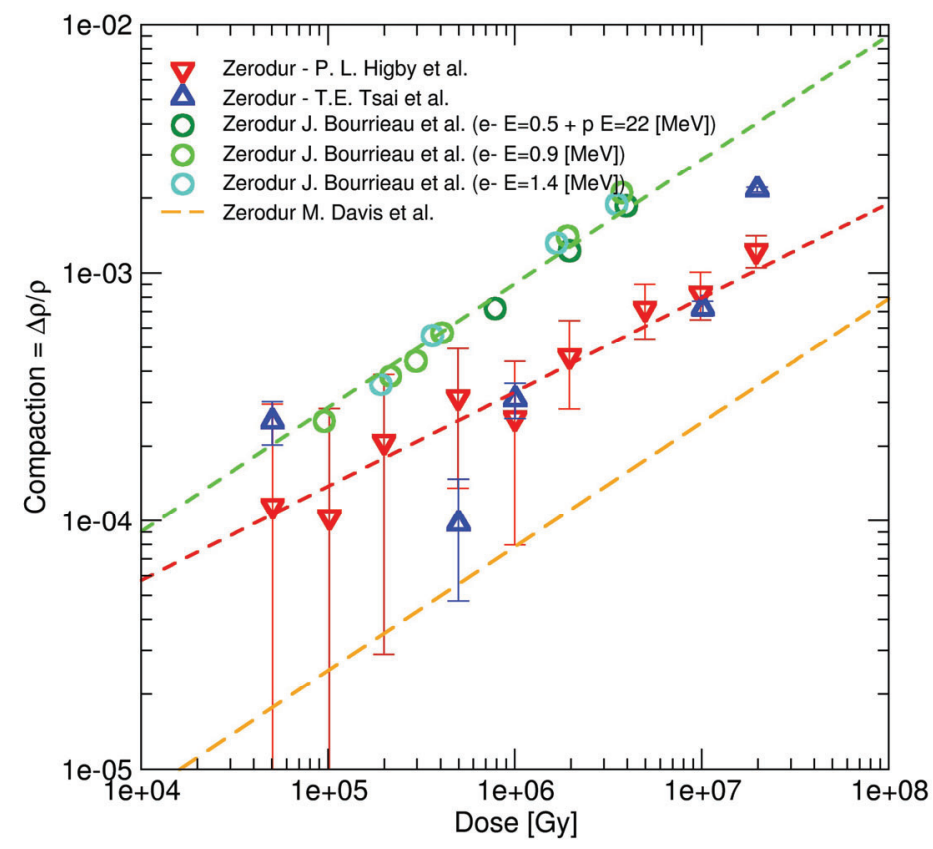

Figure 1: ZERODUR ${ }^{\circledR}$ compaction laws proposed by different authors.

It is of interest to note that the compaction properties have been investigated for large deposited doses. Indeed, all these researches vectored at the very beginning of the $80^{\prime} \mathrm{s}$ in a cold war context addressed the weapon effect, i.e. impact of very large dose deposition and high dose deposition rate. These operating conditions were far from the ones one could expect for a telescope optical mirror in a GEO orbit.

The discrepancies observed in the magnitude of the different compaction laws can be attributed to the different approaches used (density measurement, induced vertex radius of curvature measurements) as well as to the mathematical approximations used in order to describe the deformation and/or the ionizing dose deposition field. In this work, we propose an experimental methodology in order to characterize with accuracy the compaction behavior of the glass ceramic ZERODUR ${ }^{\circledR}$ under ionizing radiations. In the first section we will discuss the main working strategy. The design of the coupons is then discussed in the following section; the geometrical limitation induced by the irradiation capability (homogeneity versus dose deposition rate) will be further detailed there. In the fourth section we describe the interferometric setup designed in order to characterize the sagitta induced by the dose deposited. At last we will describe the FEM based regression approach that will be used in order to derive the parameters defining the compaction law.

\section{METROLOGICAL APPROACH}

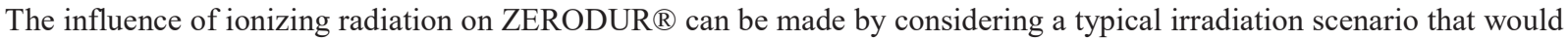
be defined for a representative orbit over a given lifetime for a particular satellite. However, this approach would be by far too specific and only of little use for the error budgeting of specific missions. Another strategy would consist in deriving a phenomenological law binding the local optical deformation to the total ionizing dose deposition that could be afterward used by the optical engineers in order to estimate their overall error budget.

Two main approaches can be considered for determining the phenomenology of the ionizing dose on the compaction of a material. The straightforward strategy consists in irradiating the material with some well-defined doses and measure the change of density observed. Albeit simple this approach can only be achieved at the required precision level ( $2 \mathrm{ppm})$ for large samples (approximatively $1002.5 \pm 2.5 \mathrm{~g}$ ), the homogeneous irradiation of such sample can be difficult and can only be achieved using gamma radiation due to its small absorbing cross section. This approach is the one chose by HIGBY, RADLEIN, however due to the smallness of the sample considered, these measures are marred with significant error bars. Another drawback of this methodology is that one does not characterize the change of optical figure observed, which is the quantity of interest in the astronomical community. Because of all these drawbacks, some authors have preferred characterizing the material compaction using an indirect optical approach, see [1][2]. Here the radiation induced sagitta of 
coupons (usually discs) were interferometrically measured. This approach supposes that the gradient of the deposited dose within the sample is well known. By combining this information with a bimetallic model, the authors were proposing a compaction law. The weakness of this approach lies in the determination of the dose profile within the samples (arbitrarily assumed to be at the edges of the solarized domain) combined with a crude bimetallic approach that simplifies a continuous dose-depth gradient into a stepwise constant function. The mathematics behind the derivations of the compaction law based on a bimetallic approach have also been debated; some misinterpretations of geometrical pre-form may have led to an overestimation of about one order of magnitude of the compaction law[5].

This work address the characterization of a compaction law by means of interferometry, the experimental measure of the radiation induced radius of curvature will be used for the fit of a phenomenological law. However, the estimation of the total ionization dose-depth within our material will be characterized by means of Monte Carlo simulations whose consistency will be cross-checked by means of Faraday cup measurements. The deformation field will not be described anymore by a thin-plate equation but by means of finite element methods in order to encompass all the possible effect that cannot be described using the Timoshenko thin plate approach (radial dependency of the strain field as well as limitation of the thin plate approximation).

\section{EXPERIMENTAL PROCEDURE}

\subsection{Coupons}

The definition of the coupon's geometry has been intensively discussed as the geometry determines many very different aspects of this Design of Experiment (DOE). First, the diameter of the coupons must be compatible with the irradiation capability of the Physikalisch-Technische Bundesanstalt (PTB) our partner irradiation facility. A large surface can be homogeneously irradiated (radially) however with a small dose deposition/rate and consequently a large coupon will require a long exposure time in order to reach the targeted peak dose along its depth. The surface figure of the coupons is a determining factor for the unequivocal derivation of the irradiation induced Vertex Radius of Curvature (VRoC). The measured effect depends on both the diameter and also on the aspect ratio of the coupons. Manufacturing aspects must be taken into account together with the irradiation ones for the definition of the coupon's geometry. Finally yet importantly, we must be able to measure properly the samples, this step also imposes some special conditions on the sample geometry. After several iteration steps, we collegially agreed in $\varnothing=50$ [mm samples having 2,0 and 5,0 mm thicknesses, these geometries were the most compatible with all the manufacturing, metrology and processing steps of this DOE.

Because of the mechanical stress induced by the irradiations, some breakage could eventually be expected at high irradiation exposure. In order to minimize a priori these occurrences, we prepared our samples by grinding with the lowest grain sized diamond CNC tool available in routine use at SCHOTT Mainz, namely D25 (front and back side of the coupons) and D20 (wall and chamfer).

The front and backsides of our samples are polished, however the walls and the chamfer will not. These latter surfaces are the most critical areas where the mechanical tension reaches its local maximum after exposure to irradiation; consequently, these areas must ideally be free of Sub Surface Damage (SSD). In order to remove these surface defect we acid etched our samples with a specifically designed etching solution (volume ratio mixture: $2 \mathrm{HF} \mathrm{38 \% :1} \mathrm{HCl} 37 \%$ : $6 \mathrm{H}_{2} \mathrm{O}$ ), during approximatively $10 \mathrm{~min}$., to this etching exposure time corresponds a material removal of about $15 \mu \mathrm{m}$, i.e. 1,5 to 3 times the assumed size of our SSDs[6]. Meanwhile, in order to prevent any surface removal of the front or back side we masked our samples using acid resistant tape, see Figure 2.

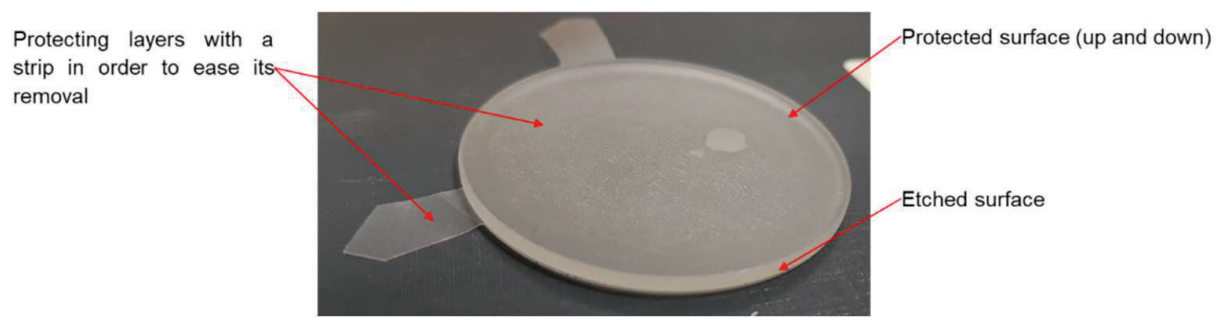

Figure 2: Etching mask of a $\varnothing=50 * 2$ mm coupon. 
The aim of this work is to characterize the change of curvature induced by the ionizing irradiation, this curvature will be interformetrically measured. This method imposes also some constraint on the manufacturing of the coupons. Interferometric interference with the polished back surface reflection will very likely occur and the question of coating the front face of our coupons has been addressed. The back face of the coupons can of course be painted but painting and cleaning several times the back face would add successive steps that would increase the risk of mishandling ${ }^{1}$. Because of this, we rejected this option. Alternately, the samples can also have a small wedge in order to displace the interfering contribution of the reflected light. However, this approach would lead to unnecessary processing complexity and because of this we also discarded it. At the end, we finally decided to deposit a simple aluminum coating of the samples, technically the coating could be very easily achieved, the total reflection induced would prevent any back reflection and would greatly ease the interpretation of the data. Moreover, the reflective aluminum coating would drain the collected e- out of the sample through the metallic mount and would prevent any electrical breakdown within our samples. In addition to this, the conductance of the electrons through the coating also increases the reliability of the measurement of the deposited charges onto the samples, since the knowledge of this quantity sharpens our estimation of the deposited dose.

\subsection{Simulations}

The determination of the compaction is a very resource-demanding task, as it requires very precise before and after irradiation metrology regarding the pieces form, as well as a very cautious irradiation procedure. In order to maximize the success of these measures, we must beforehand estimate whether the surface figure induced in our samples could be measured. In other words, we must ensure already in the preliminary phase of the Design Of Experiments (DOE) that the orders of magnitude of the expected effects are compatible with the resolution of our metrological system. This step has been bridged by means of simulations. First, we estimated the expected dose-depth in our system for different electron energies and then, we investigated the estimated change of curvature induced in this samples by means of FEM approach.

The irradiation simulations have been carried out with Geant4[7], to this end we used the version 10.3.3 of this software. The material under consideration is ZERODUR ${ }^{\circledR}$, defined using the atomic database provided by the National Institute of Standards and Technology (NIST) combined with the chemical composition of ZERODUR ${ }^{\circledR}$. The composition of surrounding material such as atmosphere, and metals involved along the irradiation line, namely titanium and stainless steel have all been described using the embedded NIST definition, namely G4_AIR, G4_TI and G4_STAINLESS-STEEL. The particle multiple scattering parameter has been defined to $1,0 \mathrm{~mm}$, a limit where we reach a numerical convergence of the results (we validated this procedure on a previous system using the same particle accelerator but using different energies).

At PTB the collimated e- beam are fired through vacuum onto a Ti sheet in order to scatter them over space. These scattered electrons are then travelling through atmosphere and will ionize the Device Under Test (DUT). The radial distribution of the collimated electrons is assumed to be normal with a full width at half maximum (FWMH $=2 \mathrm{~mm}$ ), experimental estimation provided by the PTB. This stage of the irradiation has also be encompassed in our simulation.

In order to achieve a good spatial accuracy, the target volume has been quantized into $\Delta V=\Delta x^{3}$ spatial element with $\Delta x=0.1 \mathrm{~mm}$. A sketch of these simulations is given on Figure 3 below:

\footnotetext{
${ }^{1}$ Paints are including organic binding agents that may decompose during the irradiations, the stability of the paint and the potential toxicity of the byproduct are not known. Because of this it is reasonable to assume beforehand that it may be required to paint several time the back face of the samples.
} 


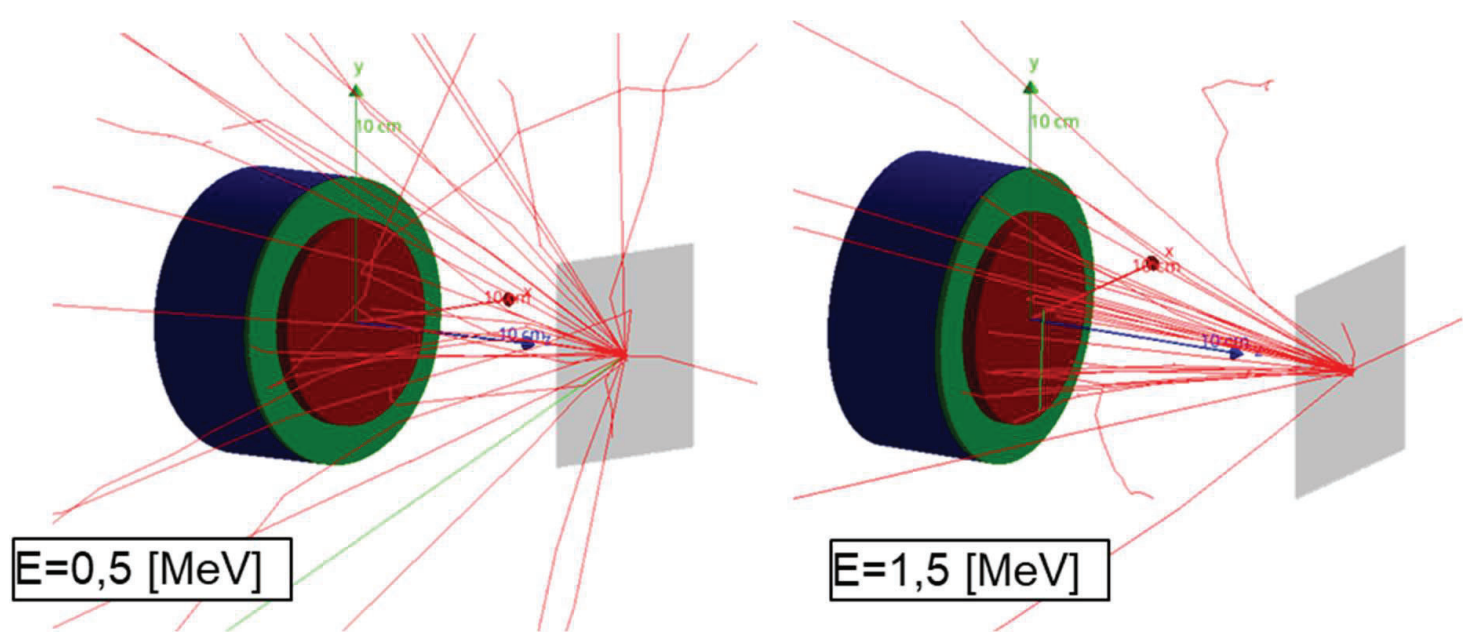

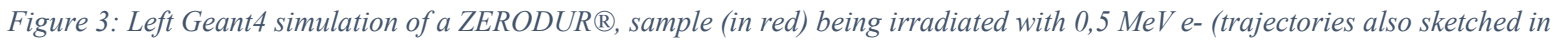
red) The collimated e- beam is scattered by a Ti sheet (in gray). Right: same irradiation setup, however the e- have this time a 1,5

MeV kinetic energy. The higher is the incident e-energy the narrower is the radial distribution of the e- on the target.

For this DOE, we aim at disentangling the effect of the dose from the effect of the e- incident energy. Historically, all the previous works dealing with compaction described this phenomenon as being exclusively depending on the dose deposited and not of the kinetic energy of the incident particles. With this work, we evaluate the validity of this assumption by irradiating our samples with two different e- incident energies.

The fraction of the e- contributing to the LEO and GEO ionizing radiations is mainly included in a [0;1.5] MeV energy range, see Figure 4, as a consequence we purposely considered energy in this range, namely $0,5 \mathrm{MeV}$ and $1,5 \mathrm{MeV}$.

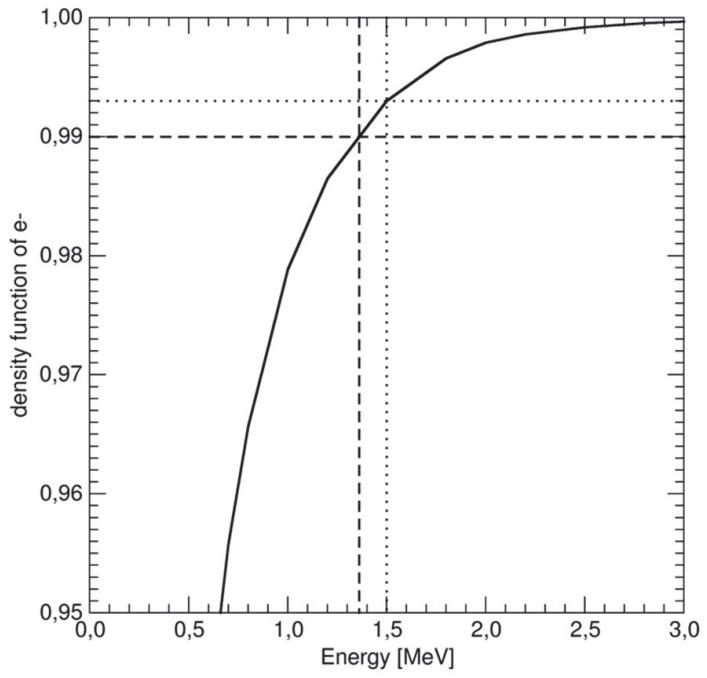

Figure 4: Cumulative distribution function of the e- energy distribution calculated for the METEOSAT10 orbit by means of SPENVIS (AE8 model) [8]. On this figure we can see that $99 \%$ of the e- over this orbit have an energy below 1,4 MeV and 99,3\% of the e-flying over this orbit have an energy below 1,5 $\mathrm{MeV}$.

The simulated dose depths at these two aforementioned energies are sketched on Figure 5 below: 


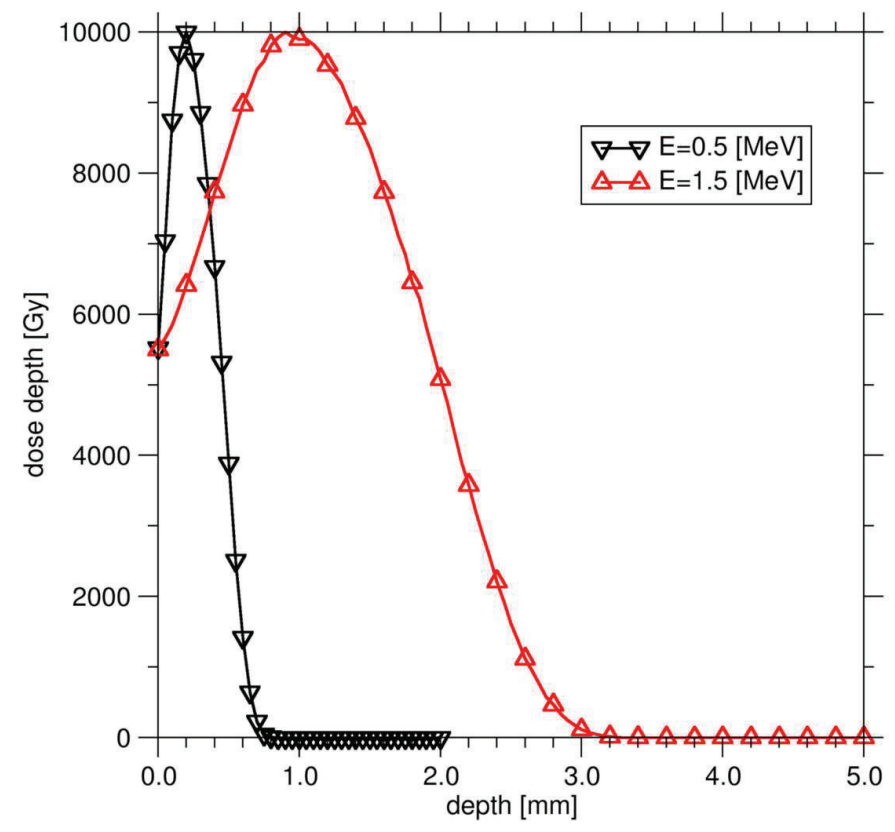

Figure 5:Dose depth profile simulated for ZERODUR ${ }^{\circledR}$, with 0,5 MeV (black down triangles) and 1,5 MeV (red up triangles) e-. The particle flux has been calibrated so that the peak dose reach the $10^{4}$ Gy value.

We considered irradiating the 2,0 mm thick samples with the $\mathrm{E}=0,5 \mathrm{MeV}$ e- beam and the $5,0 \mathrm{~mm}$ thick samples with the $\mathrm{E}=1,5 \mathrm{MeV}$ e- beam. For these energies, the penetration length of e- is about $1 / 2$ of the thickness of the corresponding coupons, the strain field is then asymmetric through the depth therefore, a significant irradiation bending is expected ${ }^{2}$. The two sketched dose depth distributions of Figure 5 have then been reinjected into a FEM model (namely ABAQUS®) software) representative of our coupons. The dose depth field has been converted into a strain field by means of a phenomenological compaction law previously determined in a joint work together with ESA ESTEC $\left({ }^{60} \mathrm{Co}\right.$ irradiation) and the PTB (density measures) ${ }^{3}$. In this FEM model we described the radiation induced stress relaxation of 2,0; and 5,0 mm thick coupons with 0,5 and $1,5 \mathrm{MeV}$.

The rough estimations of the expected deformations are given on Figure 6:

\begin{tabular}{|c|c|c|c|c|}
\hline \multicolumn{5}{|c|}{$\mathrm{E}=0,5[\mathrm{MeV}]$} \\
\hline & Dose [Gy] & $\mathrm{PV}[\mu \mathrm{m}]$ & $\mathrm{r}[\mathrm{m}]$ & nmb samples \\
\hline \multirow{4}{*}{$\bar{E}$} & $1,00 \mathrm{E}+02$ & 0,00178 & 17600 & 3 \\
\cline { 2 - 5 } & $1,00 \mathrm{E}+03$ & 0,109 & 2860 & 4 \\
\cline { 2 - 5 } & $1,00 \mathrm{E}+04$ & 0,672 & 465 & 7 \\
\cline { 2 - 5 }$\sim$ & $1,00 \mathrm{E}+05$ & 4,24 & 74 & 7 \\
\cline { 2 - 5 } & $1,00 \mathrm{E}+06$ & 25,4 & 12 & 4 \\
\cline { 2 - 5 } & $1,00 \mathrm{E}+07$ & 156 & 2 & 0 \\
\hline
\end{tabular}

\begin{tabular}{|c|c|c|c|c|}
\hline \multicolumn{5}{|c|}{$\mathrm{E}=1,5[\mathrm{MeV}]$} \\
\hline & Dose [Gy] & $\mathrm{PV}[\mu \mathrm{m}]$ & $\mathrm{r}[\mathrm{m}]$ & nmb samples \\
\hline \multirow{4}{*}{$\underset{\xi}{\varepsilon}$} & $1,00 \mathrm{E}+02$ & 0,009 & 34722 & 0 \\
\cline { 2 - 6 } & $1,00 \mathrm{E}+03$ & 0,0553 & 5651 & 3 \\
\cline { 2 - 5 } & $1,00 \mathrm{E}+04$ & 0,3404 & 918 & 4 \\
\cline { 2 - 5 } & $1,00 \mathrm{E}+05$ & 2,0915 & 149 & 7 \\
\cline { 2 - 5 } & $1,00 \mathrm{E}+06$ & 12,917 & 24 & 7 \\
\cline { 2 - 5 } & $1,00 \mathrm{E}+07$ & 79,15 & 4 & 4 \\
\hline
\end{tabular}

Figure 6: Simulated estimations of the coupon deformations for different dose peak for -left- a $50 * 2 \mathrm{~mm}$ thick coupon irradiated with ehaving a 0,5 MeV kinetic energy and -right- a 50*5mm thick coupons irradiated with e- having a 1,5 MeV kinetic energy. The expected Sagitta (SAG) is given in the Peak-to-Valley (PV) column, the corresponding concave radius of curvature in the following one. The last column corresponds to the number of samples that we allocate for these peak energy doses.

${ }^{2}$ A 3,0 mm thick disc irradiated with $1,5 \mathrm{MeV}$ would underwent a symmetric dose-distribution (symmetric with respect to the central plan). In this configuration, the strain field on the front and on the back side would partially annihilate. The resulting curvature would be small, even at high dose deposition.

${ }^{3}$ The purpose of this work is also to check the consistency of the density based measurement of the compaction law with the measurement based on the interferometric approach. This topic will be the subject of a future communication. 
For both energies, the total dose deposited will be modulated by changing the radiation exposure time keeping all other parameters constant (positioning, e- firing frequency, etc...).

Remember that the deformation listed in the above table is only a guideline in order to estimate the orders of magnitude of the expected deformation. These values solely indicate whether the induced sagitta could be interferometrically measured with confidence and thus be helpful to us populating our DOE.

The internal stress fields expected in for a dose deposition peak of $10^{7}$ Gy have also been simulated see Figure 7:

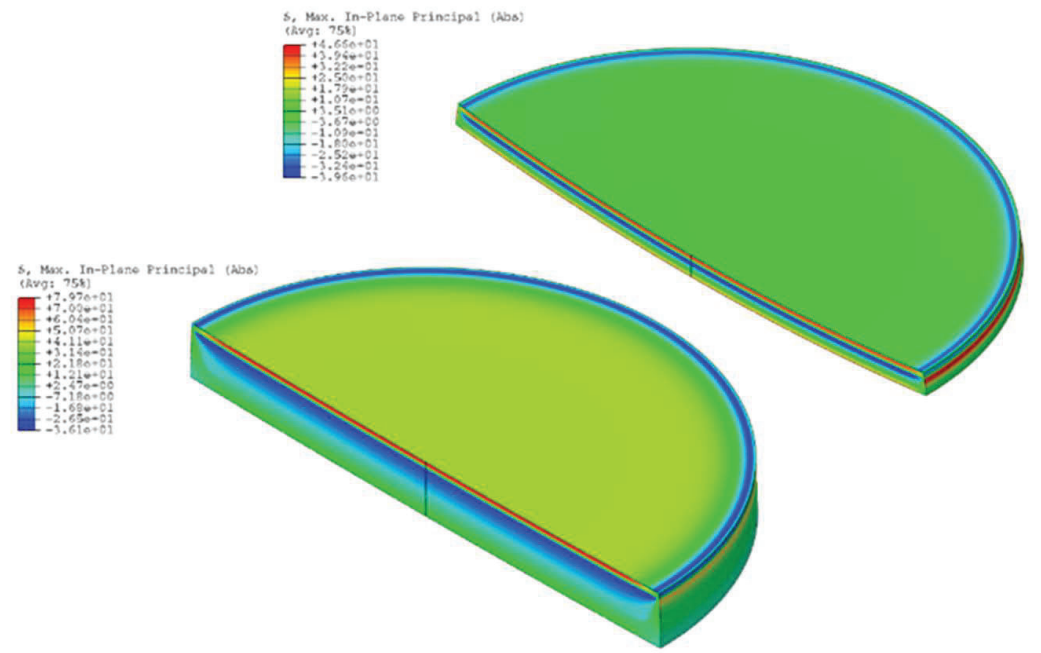

Figure 7: Internal stress expected into a -bottom- $5 \mathrm{~mm}\left(E_{e_{-}}=1,5 \mathrm{MeV}\right)$ and -top $-2 \mathrm{~mm}\left(E_{e_{-}}=0,5 \mathrm{MeV}\right)$ back sample for a dose peak of about $10^{7} \mathrm{~Gy}$.

These stress estimations results made necessary removing the SSDs at the machining stage in order to mitigate any potential breakage. We clarify that these peak doses over these deposition length scales are not representative at all of any typical electron dose-depth profile over any traditional orbit. The corresponding induced curvature listed on Figure 6 are not representative of any deformations that one may expect over any space orbit. These coupons have been exclusively designed in order to characterize a compaction law for those who want to calculate the estimated deformation of their particular mirror design over their hypothesized irradiation scenario, which is depending on the satellite shielding, on the orbit, solar events as well as mission lifetime.

\subsection{Experimental e- irradiation flux characterization}

The coupons under consideration have been designed for a 0,5 and 1,5 MeV e- energy. The energy of the incident e- is an aspect that will define the dose-depth profile. Practically, the e- are collimated by magnetic field within the accelerator, then the collimated beam is scattered by a $50 \mu \mathrm{m}$ thick Ti sheet. The scattered beam is not radially homogeneous but rather follows a normal distribution $f(r ; z)$ :

$$
f(r ; z)=N e^{\left(-\frac{r^{2}}{2 \sigma(z)^{2}}\right)}
$$

Where $N$ is a normalization factor, $\sigma(z)$ correspond to the standard deviation which is a function of the distance $z$ between the scattering Ti sheet and the target, but also a parametric function of the thickness of the scattering metal sheet and the energy of the incident e- collimated in the beam. In order to characterize at best the dose-profile within our coupons (over the thickness but also radially) it is crucial to determine with accuracy the characteristics of the e- flux (in other words $\sigma(z)$ ). The detectors typically used for this task at the PTB (PTW Octavius 15000) regrettably are not sensitive enough for the energy considered. Since the penetration depth of e- is small, we are out of its sensitivity domain. In order to circumvent these difficulties, we performed a Geant4 Monte Carlo simulations of the irradiation line to estimate the expected radial distribution of the beam corresponding to the energy considered. 
Based on these simulations, we were able to propose a first guess regarding the distribution: that for a $500 \mathrm{keV}$ energy we may expect a $90 \%$ radial homogeneity field over our coupons at a distance $z \approx 150 \mathrm{~mm}$.

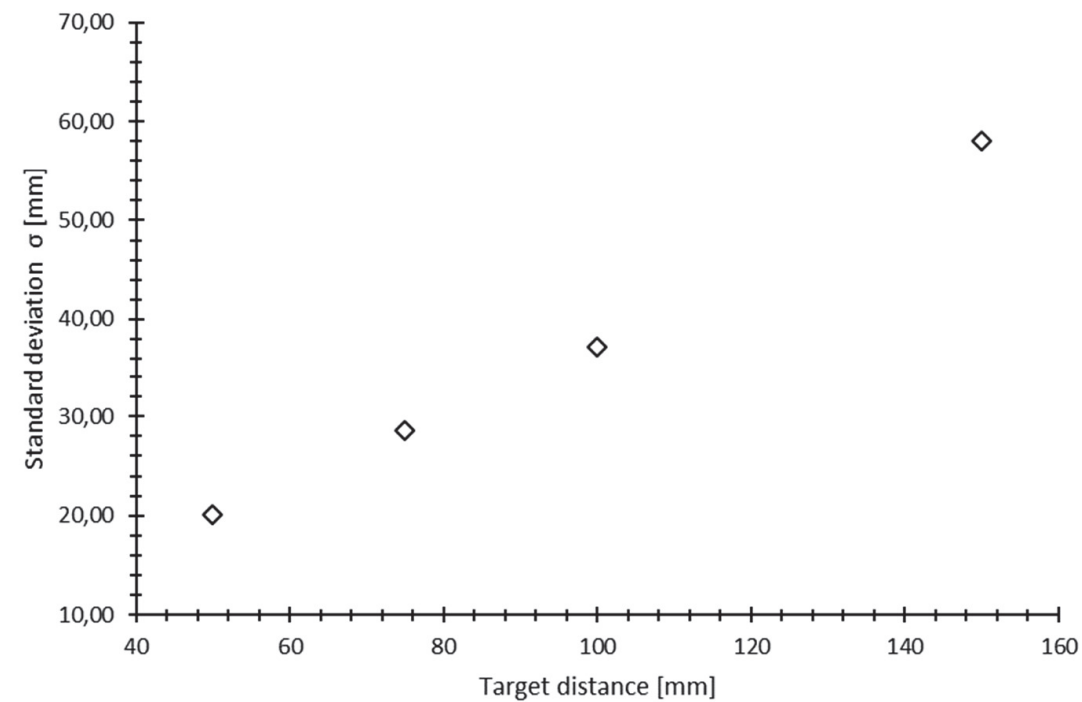

Figure 8: Estimation of the expected standard deviation of the electron radial distribution for different DUT distances from the source for incident electron energies of $500 \mathrm{keV}$. At a distance of $z=150 \mathrm{~mm}$ we expect a $\sigma$ value of about $58 \mathrm{~mm}$. To this value correponds a homogeneity of the electron flux of about $91 \%\left(=e^{-\frac{25^{2}}{258^{2}}}\right)$.

Since these estimations are purely based on computational approaches, they must be cross-checked. To this end, we designed very simple Faraday cups having different radii, namely $R=15 ; 25 ; 40 ; 60 \mathrm{~mm}$, see Figure 9:

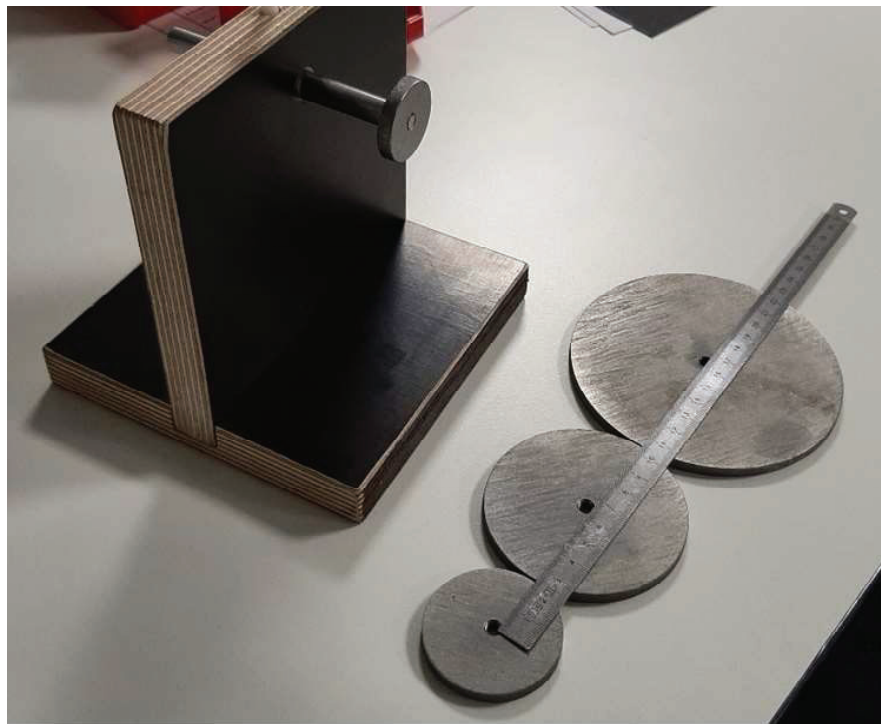

Figure 9: Faraday cup used for the characterization of the e- beam at 0,5 $\mathrm{MeV}$ and 1,5 $\mathrm{MeV}$

Some preliminary beam characterization tests will be carried out with these cups placed at a $z=150$ mm distance from the canon with $500 \mathrm{keV}$ e- incident electrons. The different charges collected for different disc radius keeping all the other 
irradiation parameter constant will give access to the experimental electron distribution. Practically, the charge collected on a metal discs of radius $R$ at a distance $z$ can be formally written $C_{R}^{Z}$ :

$$
C_{R}^{Z}=\int_{\theta=0}^{\theta=2 \pi} \int_{r=0}^{r=R} f(r ; z) \cdot d S
$$

With $d S=r . d r . d \theta$ being a surface element (we took the usual cylindrical coordinates in order to describe our setup).

If we now further develop the above expression we obtain:

$$
C_{R}^{Z}=\int_{\theta=0}^{\theta=2 \pi} \int_{r=0}^{r=R} f(r ; z) \cdot d S=\int_{\theta=0}^{\theta=2 \pi} \int_{r=0}^{r=R} N e^{\left(-\frac{r^{2}}{2 \sigma(z)^{2}}\right)} r d r d \theta
$$

This above integral can be further simplified into:

$$
C_{R}^{Z}=N * 2 \pi * \sigma(z)^{2} *\left[1-e^{\left(-\frac{R^{2}}{2 \sigma(z)^{2}}\right)}\right]
$$

The first term of this expression, $N * 2 \pi * \sigma(z)^{2}$ corresponds to the total amount of e- fired on the beam line during the test. Per design, this value will be kept constant over our experimentation as we will proposedly investigate all the different Faraday discs using the same irradiation parameters (i.e. also the total charge of fired e- per disc). Considering this, we can rewrite the expression of the collected charge:

$$
C_{R}^{Z}=C_{0} *\left[1-e^{\left(-\frac{R^{2}}{2 \sigma(z)^{2}}\right)}\right]
$$

With $C_{0}$ being the e- charge fired per test. The value $C_{0}$ can be experimentally determined using the ICT (Bergoz Instrumentation) at the end of the beam line. Using all these measured charges we can then reconstruct the value $\sigma(z)$ that characterizes the beam using either the ICT value and/or using different cup radius. The consistency of these measurements with the simulated collected charge fraction at a distance of $150 \mathrm{~mm}$ from the beam source for $500 \mathrm{keV}$ electron energies presented on Figure 10 will validate our simulation approach as well as the hypothesized dose-depth distribution.

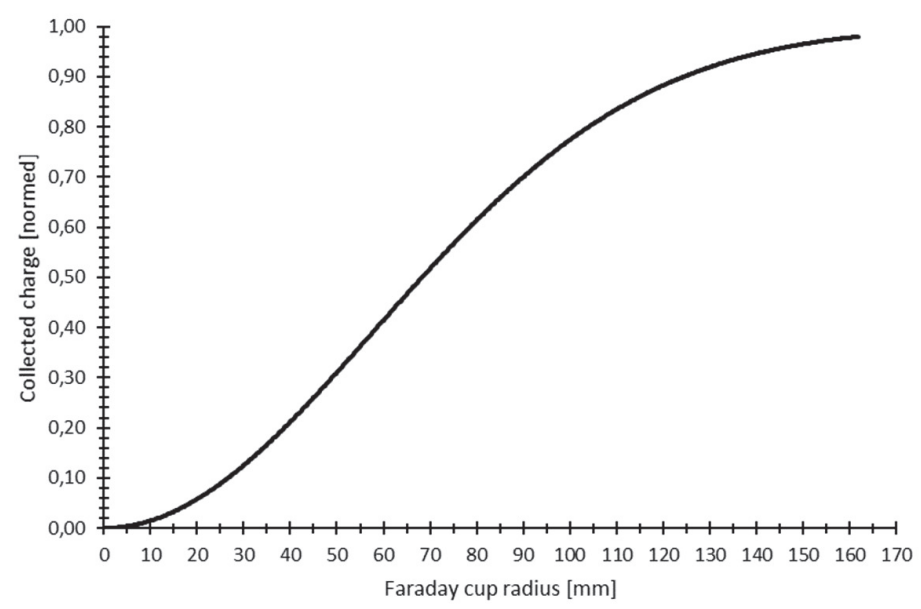

Figure 10: Expected electron fraction collected onto a cylindrical Faraday at a distance $z=150 \mathrm{~mm}$ for an incident electron beam having a $500 \mathrm{keV}$ kinetic energy. 


\subsection{Faraday mounting cup}

The coupons will be held in a mount specially designed for the purpose of our experiments. This mount is made with a metal backplate and a metallic cover ring. The ZERODUR ${ }^{\circledR}$ samples will be loosely staked between the backplate and ring. The manufacturing tolerance of the cover ring have been precisely defined in order to minimize the surface masking but still preventing the coupons from falling. Furthermore, the small overlapping area will ensure the electrical contact between the coupons aluminum coating and the mount. The mount will be connected to a Coulombmeter for the in-situ monitoring of the irradiation experiment.

The design of this Faraday cup is sketched on the Figure 11 below:

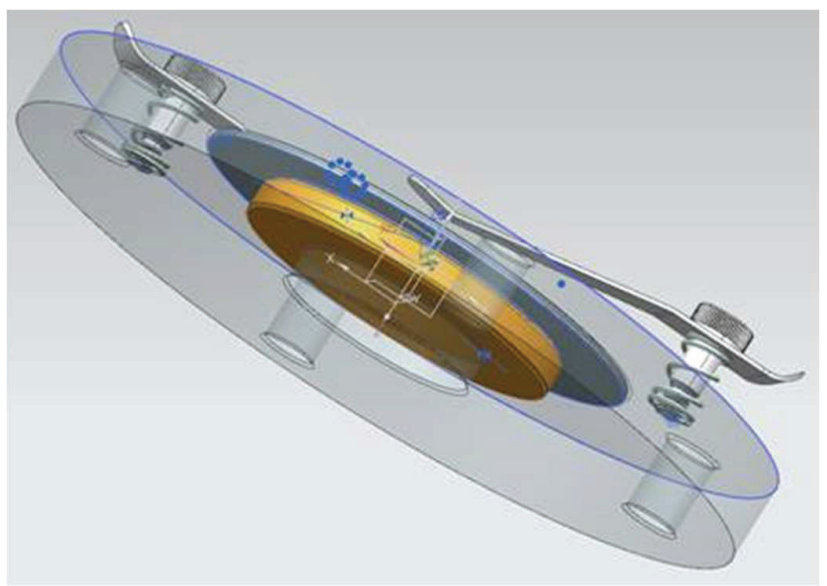

Figure 11: Technical design of the sample holder. The sample is pictured in yellow; the metal holder is pictured in gray (front ring) or in transparent gray (background). This mount will be connected to a Coulombmeter in order to estimate the amount of e-collected on the target during the experiment.

The Faraday cups used for the irradiations are pictured on Figure 12.

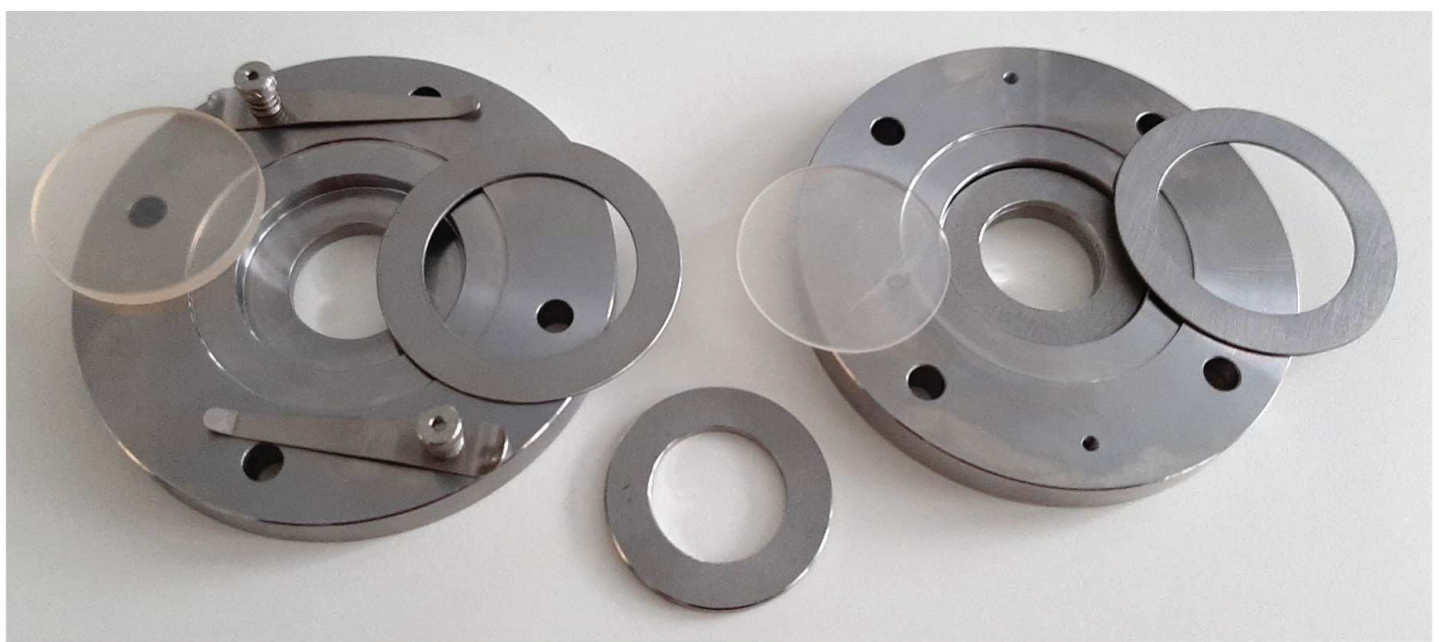

Figure 12: Faraday cups based on the design presented on Figure 11.

\section{ANALYTICAL PROCEDURE}

Evaluation of mirror surface radiation compaction at moderate commonly experienced in-orbit radiation doses requires substantial coupon metrology precision, and repeatability of the metrology setup. Measurement precisions of a few 
nanometers are required to address the expected magnitudes. Arizona Optical Systems (AOS) has established a surface metrology configuration to measure coupons using a HeNe $(\lambda=623.8-\mathrm{nm})$ Zygo Fizeau interferometer with a horizontal $150 \mathrm{~mm}$ beam placed $150 \mathrm{~mm}$ away from the coupon. Measurements are to be made before and after irradiation and compared, imposing that the interferometer and reference flat must maintained in stable hardware and software configurations covering this interval. Precautions for high accuracy surface metrology are imposed, including temperature control $( \pm 0.5-C)$ under laminar flow and vibration isolation. Measurements are made to a defined procedure, and to further ensure consistency, the same engineer makes the before and after irradiation measurements.

The coupon is held in a Delrin fixture, ensuring that the part is perpendicular to the metrology beam, and marks establish azimuthal clocking of the coupon's surface. Preliminary tests of surface measurement repeatability were made to qualify the process with on-hand, partially representative, coupons exhibiting a relatively crude surface accuracy $\sim 320 \mathrm{~nm}$ PV, or $\sim \lambda / 2$. Even with this large value of surface error, repeatability was $2.6-\mathrm{nm}$ before and after removing and replacing the coupon, and 4.5-nm repeating the measurement decentered on the beam. Multiple measurements are made before and after, averaged for precision. Since the actual coupons to be irradiated are substantial better ( $\sim \lambda / 6$ or $\sim 105-\mathrm{nm}$ for the 2$\mathrm{mm}$ thick coupons, $\sim \lambda / 20$ or $32-\mathrm{nm}$ for the $5-\mathrm{mm}$ thick coupons), the repeatability, and ability to discern changes, is expected to be as much as $3 \mathrm{x}$ to $10 \mathrm{x}$ better than the evaluative result on the process qualification samples. Control samples, having not undergone irradiation, will accompany those irradiated, and be re-measured with these radiation exposed samples to establish the metrology uncertainty.

\section{COMPACTION LAW DERIVATION}

\subsection{Strategy}

Previous determination of the compaction using optical approaches were based on a rough estimation of the dose profile (LiMi estimation of the darkening limit of the glass) further coarsened by means of a bimetal analogy analysis. In order to catch most of the phenomenology of the compaction effect, we will proceed to the derivation of the dose depending phenomenological law by means of a least square algorithm. In this approach, we will minimize the discrepancies observed between the experimentally measured VRoC of our coupons and the numerically forecasted one by varying the parameters defining the phenomenological compaction law used into the FEM software. The Chi-squared function driving the fitting of the compaction law is defined:

$$
\chi^{2}=\frac{1}{N} \sum_{i=1 . . N}\left[\operatorname{Pow}_{i}^{e x p}-\operatorname{Pow}_{i}^{\text {sim }}\left(\left\{A_{p} ; B_{p}\right\}, D_{i}(z)\right)\right]^{2}
$$

With:

- $\quad N$ : Being the number of samples into consideration;

- $\quad i$ : an index denominating a given sample;

- Pow: meaning the estimated power or VRoC;

- The superscript exp and sim designate the origin of the data, respectively experimental or simulated;

- The doublet in brace $\left\{A_{p} ; B_{p}\right\}$ corresponds respectively to the prefactor and exponent of the power law used for describing the compaction, $\frac{\Delta \rho}{\rho}(z)=A D(z)^{B}$ with $D(z)$ the dose deposited at a given depth $z$. The subscript $p$ indicates the iteration level of the algorithm.

Being based on FEM simulations, this procedure offers many advantages, i.e. we will consider our material mostly as a continuum and not as a composite. Moreover, we will not rely on the thin plate approximation whose validity depends on the radius/thickness ratio. The use of FEM may comprehensively address issues connected to some radial dependent dose distribution (remember that the mounting ring will shield the borders of the coupons).

The correctness of the least squares algorithm has been tested on a model system where the experimental powers values have been substituted with FEM based values - reference data. We then started the least-square minimization algorithm using phenomenological compaction parameters, which were significantly different from the reference data. The graphical results summarizing the whole optimization process are provided on Figure 13. 


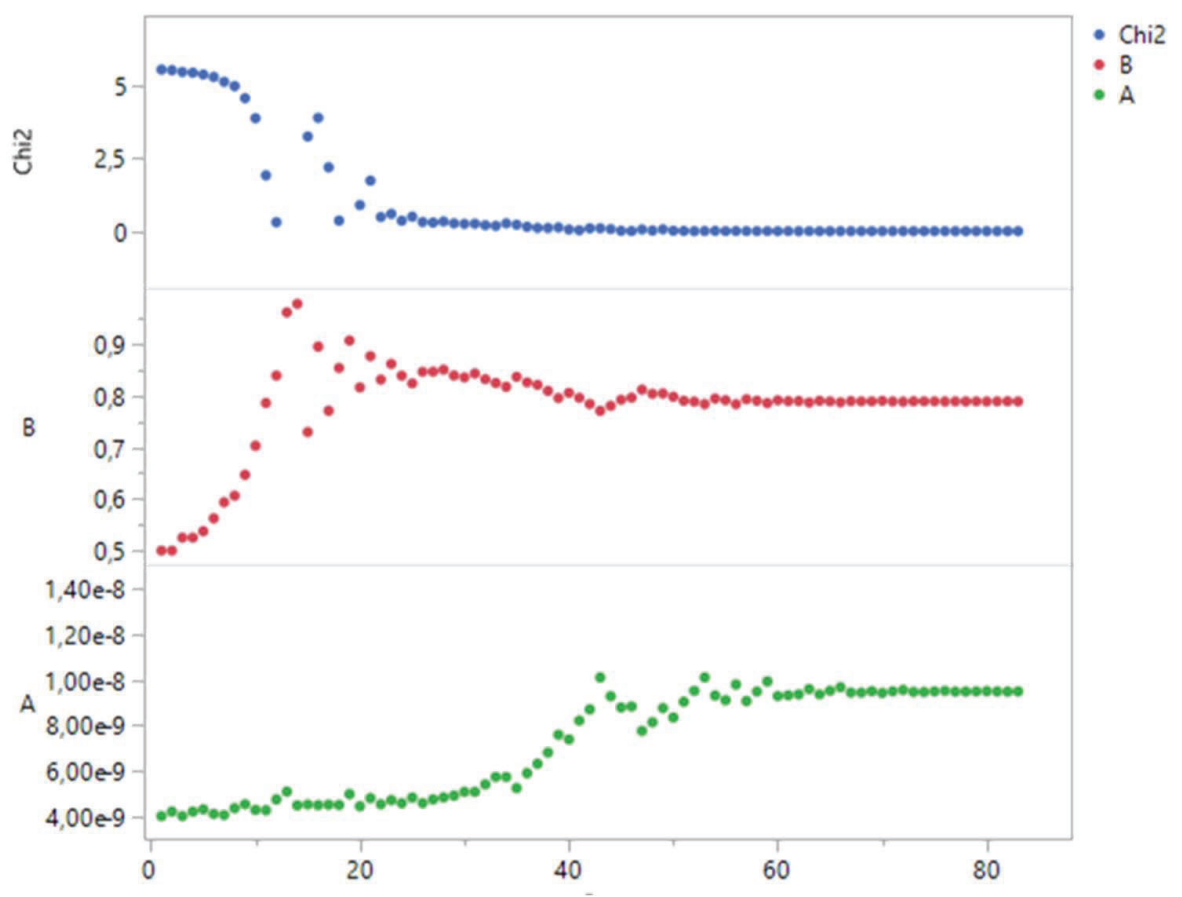

Figure 13: Top-blue: $\chi^{2}$ evolution over the iterations. Middle-red:estimation of the exponent $B$ as a function of the iteration level, Bottom green: estimation of the compaction prefactor $A$ as a function of the iteration level.

\subsection{Power characterization}

The irradiations profile being mostly depth dependent and marginally radius depends we are mainly expecting an additional power contribution to the surface shape of our coupons. This power contribution can be characterized by means of the Zernike polynomial decomposition available on the interferometric software solution. In our present case, the coefficient of interest is the defocus term (radial degree $n=2$, azimuthal degree $m=0$, OSA/ANSI index $j=4$ ):

$$
Z_{2}^{0}(\rho)=\sqrt{3}\left(2 \rho^{2}-1\right)
$$

Where $\rho$ stands for the normed radius of our optic, i.e. $\rho \in[0 ; 1]$. Formally, the Zernike approach aims at describing the surface profile $G(\rho, \varphi)$ of an optic as being the outcome of an infinite sum of Zernike factor:

$$
G(\rho, \varphi)=\sum_{m, n}\left(a_{m, n} Z_{n}^{m}(\rho, \varphi)+b_{m, n} Z_{n}^{-m}(\rho, \varphi)\right)
$$

Where $a_{m, n}$ and $b_{m, n}$ are the weighting factors indicating the magnitude of the respective Zernike contributions and $\varphi$ is the polar angle (polar coordinate system):

$$
a_{m, n}=\frac{2 n+2}{\varepsilon_{m} \pi} \iint_{\varphi=\rho=0}^{\rho=1, \varphi=2 \pi} G(\rho, \varphi) Z_{n}^{m}(\rho, \varphi) \rho d \rho d \varphi
$$

Therefore, the $a_{0,2}$ term is an indication of the magnitude of the optical power of our mirror. Formally, we can further refine our understanding of this term by performing a change of variables from cylindrical to Cartesian coordinates:

$$
\begin{aligned}
& a_{0,2}=\frac{2}{\pi} \iint_{\varphi=\rho=0}^{\rho=1, \varphi=2 \pi} G(\rho, \varphi) \sqrt{3}\left(2 \rho^{2}-1\right) \rho d \rho d \varphi \\
& a_{0,2}=\frac{2}{\pi} \iint_{x, y} G(x, y) \sqrt{3}\left(2\left(x^{2}+y^{2}\right)-1\right) \mathrm{d} x d y
\end{aligned}
$$


For a surface shape mostly dominated by a spherical curvature term, we have a local elevation $G$ following:

$$
G(\rho, \varphi) \approx \frac{(\rho r)^{2}}{2 R}
$$

With $R$ being the VRoC of the optic, $r$ the radius of the optic and $\rho$ the local radial position. In this configuration we can see that the contribution to the defocus term scales like $1 / R$ :

$$
a_{0,2}=\frac{2}{\pi} \iint_{\varphi=\rho=0}^{\rho=1, \varphi=2 \pi} G(\rho, \varphi) \sqrt{3}\left(2 \rho^{2}-1\right) \rho \mathrm{d} \rho d \varphi=\frac{r^{2}}{8 \sqrt{3} R}
$$

By means of the Zernike decomposition, we can easily access to the radius of curvature or to the overall sagitta (SAG = $\left.r^{2} / 2 R\right)$, these quantities can easily be derived from FEM structure simulations and will be used for the calculation of the merit function.

\subsection{Exclusive contribution of the irradiation to the change of $\mathrm{VRoC}$}

Prior to the irradiation experiments, we expect some remnant polishing patterns and consequently some power profiles prior to any irradiation procedure. The characterization of the compaction is based on the characterization of the radiation induced power, this means that we must properly disentangle the power contribution due to the coupon polishing and the power contribution due to irradiation from the overall measured power contribution.

Formally, the spherical contribution to the surface deformation can be written:

$$
z=R-\sqrt{R^{2}+(r \rho)^{2}}
$$

If we know assume that the $\operatorname{VRoC}(R)$ is very large compared to the radius of the optic $(r)$ we can approximate the above expression using a $1^{\text {st }}$ order Taylor expansion into:

$$
z=\frac{(\rho r)^{2}}{2 R}
$$

If we now assume that the compaction is superimposing an additional contribution to the curvature we get:

With

$$
\begin{gathered}
z=\frac{(\rho r)^{2}}{2 R_{\text {polishing }}}+\frac{(\rho r)^{2}}{2 R_{\text {compaction }}} \\
z=\frac{(\rho r)^{2}}{2 R_{\text {pol. }+ \text { comp }} .}
\end{gathered}
$$

$$
R_{\text {pol. }+ \text { comp. }}=\frac{R_{\text {polishing }} R_{\text {compaction }}}{R_{\text {polishing }}+R_{\text {compaction }}}
$$

The Zernike decomposition of experimental interferograms prior to the irradiation runs will provide the VRoC attributed to the polishing quality $\left(R_{\text {polishing }}\right)$, whereas the Zernike defocus contribution measured after irradiation will provide information about the cumulated contributions of the surface quality and irradiation induced compaction of the surface $\left(R_{\text {pol. }+ \text { com }}\right.$.). From these two experimental quantities, we can then derive the exclusive contribution of the compaction to the overall VRoC following:

$$
R_{\text {compaction }}=\frac{R_{\text {polishing }} R_{\text {pol. }+ \text { comp. }}}{R_{\text {polishing }}-R_{\text {pol.tcomp. }}}
$$

\section{CONCLUSIONS AND PERSPECTIVES}

The measure of the compaction of a material is a complex task, this characterization can be made using two very different paths, either using the direct measurement of the density or using an indirect optical approach. The indirect measure of the density is by far the most sensitive strategy; however, it requires a deep understanding of the working hypotheses that bind the experimentally observed optical deformation to the phenomenological compaction law. In this publication, we show all the preparatory work readied for the determination of the compaction law by means of precise optical interferometry. This work will now enter in its final phase during the first half of 2021, samples prior to the irradiation will be measured as soon as a free irradiation slot at PTB will be available. Controlled interferometric measurements prior and after irradiations will be separated by a short time span in order to guarantee the stability of the metrological chain. 


\section{REFERENCES}

[1] Bourrieau, J., Roméro, M., ESA Spacecraft Mater. In Space Environment, 275-285 (1979).

[2] Bourrieau, J., et al, « Étude expérimentale sur l'irradiation par les particules à haute energie des miroirs utilisés dans le télescope radiomètre METEOSAT », Report N²145/74/CG (1974).

[3] Tsai, T., E., Higby, P., L., Friebele, E., J., Griscom, D. L., J. Appl. Phys. (62), 3488-3490 (1987).

[4] Higby, P., L., Friebele, E., J., Shaw, C., M., Rajaram, M., Graham,E. K., Kinser, D. L., Wolff, E. G., J. Am. Ceram. Soc. (71), 796-802 (1988).

[5] Davis, M., J., Fainberg, J., Proceedings of SPIE, Optical Materials and Structures Technologies, (5179), 38-49, (2003).

[6] Leys, A., Hull, T, Carré, A., Westerhoff, T., ICSO 2020 Paper session 250.

[7] Allison, J. et al, Nuclear Instruments and Methods in Physics Research Section A: Accelerators, Spectrometers, Detectors and Associated Equipment, (835), 186-225 (2016).

[8] SPENVIS - "Space Environment Information System", https://www.spenvis.oma.be/credits.php hosted by the Belgian Federal Science Policy Office. 